\title{
Sur la stabilité des volcans et autres terrils
}

\section{P. DUFFAUT}

Expert et Consultant en Génie géologique

130, rue de Rennes

75006 Paris

pduffaut@compuserve.com
Comme les volcans, certains tas et terrils sont construits en matériaux tombés verticalement d'un point au-dessus d'eux, ce qui explique leur même forme conique. Les ruptures de tas et digues de stériles font partie des risques industriels et les accidents recensés depuis quelques décennies ont conduit à une réglementation de plus en plus stricte. L'exemple du mont Ontake (Japon) en 1986 a montré que parmi les risques qui menacent les alentours des volcans, certains sont indépendants de l'activité volcanique et tiennent seulement à des structures internes favorables aux glissements de terrain. Comme tout relief ou tas est, ou peut devenir, un château d'eau, toute pente est, ou peut devenir, un barrage pour l'eau qui est à l'intérieur. Tous sont menacés par la pression d'écoulement de cette eau, et les tas coniques davantage que d'autres reliefs en raison de la position des contrastes de perméabilité suivant des surfaces parallèles aux pentes extérieures. Contrairement aux barrages, les volcans sont construits au hasard sur des terrains non préparés ; comme les dunes, les moraines, et les cônes d'éboulis et d'alluvions, ils appartiennent à la famille des reliefs construits et non à celle des reliefs creusés par l'érosion. Ainsi les volcans menacent les populations qui vivent à leurs pieds, même lorsqu'ils sont éteints depuis longtemps.

Mots-clés : volcan, terril, érosion, eau souterraine, stabilité des pentes.

\section{On stability of volcanoes and other heaps}

Just as volcanoes, some heaps of aggregate on mine refuse are built with materiais fallen from one point over them, which explains their common cone shape. The failures of tailings dams and other embankments are classified among industrial risks, and events that occurred within the last decades have led to more and more stringent rules. The example of Mount Ontake, Japan, in 1984 showed that, among hazards around volcanoes, some do not result from volcanic activity, but only from internal structures favourable to landslides. As any natural mound or unnatural heap is or may become a water tower, any slope is or may become a dam retaining the water inside. All of them are threatened by the filtration pressure of water, and the cone heaps more than other summits, due to permeability contrasts along the surfaces parallel to the slopes. Contrary to dams, volcanoes are built at random on unprepared grounds; as well as dunes, moraines, debris and alluvium cones and deltas, volcanoes belong to the family of built up summits, instead of most of slopes which have been carved by erosion. Volcanoes threaten people living at their feet, even when they are long since extinct.

Key words : volcano, refuse heaps, erosion, groundwater, slope stability. 


\section{Introduction}

La parenté de forme frappe les visiteurs du « plat pays n du Pas-de-Calais ou de la ville de Saint-Étienne : les terrils sont pris pour des volcans (Figs. 1 et 2) ! Ce n'est pas un hasard, les uns et les autres sont des tas de matériaux tombés (ou « retombés $»$ ) d'en haut, dont les éléments plus ou moins disparates forment des cones plus ou moins réguliers ; on trouve aussi des tas de granulats coniques sur le carreau des carrières. En vérité les formes géométriques pures sont assez rares dans la nature pour que celles-ci soient jugées remarquables. Outre ces cônes, d'autres dépôts de matériaux comme les décharges et les terrils en « verses $»$, ont des faces planes, comme aussi les remblais des barrages et les digues de canaux ; leurs formes les signalent à l'observateur comme anthropiques, alors que les nappes d'éboulis au pied des falaises, par exemple, ont des formes beaucoup moins réguliéres.

Les accidents par rupture de terrils font partie des risques industriels (Jupille, Belgique, 3 février 1961 ; Aberfan, pays de Galles, 21 octobre 1966; Stava, Italie du Nord, 19 juillet 1985) même lorsqu'ils sont déclenchés par des séismes majeurs (Chili 1965). Le cas particulier des barrages de stériles implique l'eau, même si elle n'est pas toujours bien visible, les autres cas aussi, en général.

Le glissement catastrophique du volcan japonais Ontake, le 14 septembre 1984 (Duffaut, 1987) montre que les pentes des volcans sont particulièrement

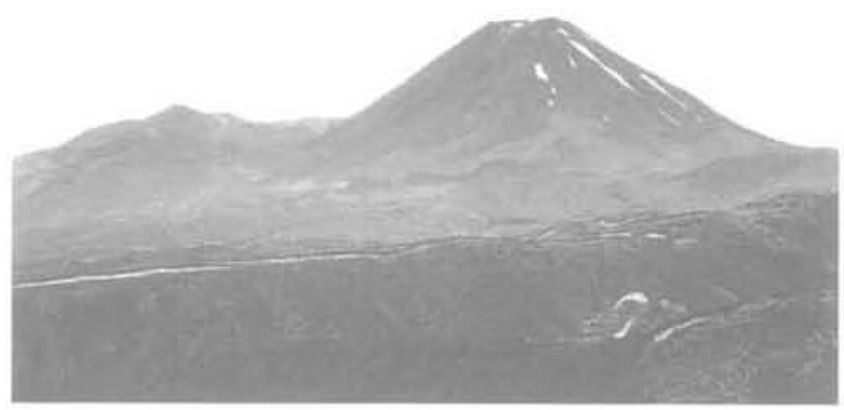

FIG.1 Le volcan Tangariro; Nouvelle-Zélande (photo Pierre Duffaut). Volcano Tangariro, New Zealand.

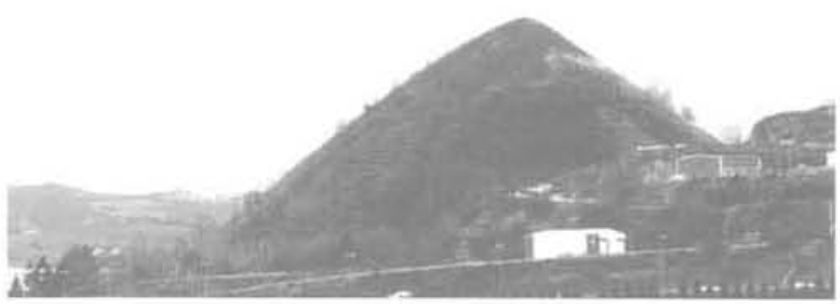

FIG. 2 Le terril du Soleil à Saint-Étienne (photo Yves Paquette, INERIS).

Soleil mine refuse heap, in Saint Etienne city, France. sujettes aux glissements de terrain, même en dehors de toute manifestation du volcanisme ; ainsi presque tous les volcans présentent des cicatrices de glissements de terrains, soulignées par la neige sur la figure 1. L'article est donc consacré aux caractères communs aux terrils anthropiques et aux volcans, que l'on doit considérer comme des terrils volcaniques.

\section{2}

\section{L'eau et la stabilité des pentes (rappels)}

L'eau courante érode la surface des pentes grâce à sa force vive, la moindre pluie ravine les sols nus et surtout les remblais et terrils dont la cohésion est en général moindre en surface que celle des sols naturels. Si l'érosion interne est moins rapide que l'érosion superficielle (et moins visible), elle n'est pas moins dangereuse, comme le montrent la théorie et le comportement des barrages en remblai; la force vive est alors négligeable, mais l'écoulement transmet au solide poreux une pression de courant.

Tout relief est un château d'eau, au moins potentiel ; donc toute pente est ou peut devenir un barrage (Duffaut et Louis, 1972 ; Duffaut, 1992) ; la pluie qui tombe sur un tas de sable transporte les fines vers l'extérieur et colmate le pied du talus; la pluie suivante s'écoule moins bien; plus la pluie est intense, plus vite et plus haut le tas se remplit; un jour le tas s'affaisse ou « crève $)$. Ainsi tous les talus artificiels comme tous les versants naturels sont menacés par la montée de l'eau souterraine, surtout par les montées les plus rapides; mais seuls les barrages sont précisément construits pour lui résister (Duffaut, 1994).

La pente d'un volcan peut être schématisée par des couches parallèles de perméabilités différentes (Fig. 3) ; la pression d'écoulement se concentre, surtout en régime transitoire, sur les surfaces de contraste (dans le sens de plus à moins perméable). Il y a donc lieu de comparer l'intensité de la pluie et la perméabilité des couches les moins perméables: l'intensité d'une pluie

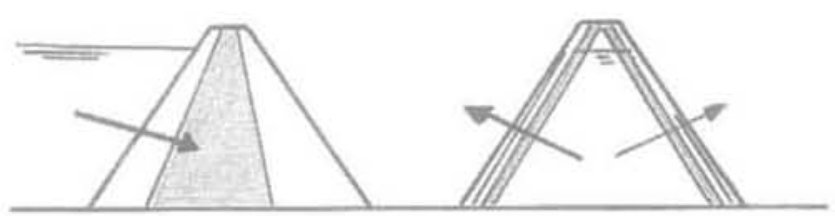

Fig. 3 Coupes schématiques comparées d'un volcan, et d'un barrage en remblai ; l'eau extérieure exerce une poussée dirigée vers le bas sur le noyau “étanche » placé au centre du barrage, alors que l'eau intérieure exerce une poussée vers le haut sur des couches "étanches » proches des pentes du volcan (échelles différentes).

Compared schematic cross-sections of a volcano and a fill dam; the thrust of outside water against the central impervious core of the dam is directed downwards, while the thrust of inside water acting against the impervious layers close to the volcano slopes is directed upwards (not at the same scale). 
violente, par exemple $36 \mathrm{~mm}$ en une heure, s'exprime aussi bien par $10^{-5} \mathrm{~m} / \mathrm{s}$; transposée en perméabilité, c'est une valeur plutôt moyenne, qui peut convenir à des couches de cinérites ; mais en cas d'altération argileuse de ces cendres, leur perméabilité sera divisée par 1000 ou davantage. Alors l'effet d'une pluie violente soulèvera facilement la partie superficielle du versant. Le raisonnement suppose que le sommet n'est pas étanche pour que la pluie pénètre. Lorsqu'on considère des surfaces de glissement plus profondes, il faut cumuler l'effet des pluies sur une certaine durée, qui peut atteindre plusieurs semaines.
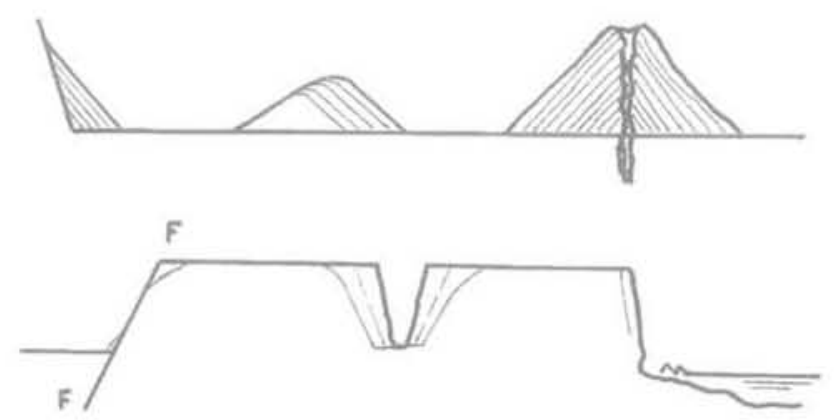

HQ.4 Coupes schématiques de pentes construites, talus d'éboulis, dune, volcan, et de pentes découpées ou " sculptées ", falaise marine et gorge d'un cours d'eau (d'après Duffaut, 1994), plus l'escarpement de faille qui n'appartient à aucune de ces catégories (échelles différentes).

Schematic cross-sections of built slopes, talus, dune, volcano, and of carved slopes, sea bluff and river gorge, plus a fault escarpment which does not belong to any of these categories (not at the same scale).

Bien entendu les versants les plus raides et les plus hauts sont les plus menacés. Les volcans boucliers, à pentes faibles, ne montrent guère les cicatrices visibles sur la plupart des autres, par exemple sur la photo classique du Fuji-Yama. Les coulées de laves ont aussi un pouvoir érosif, certains ravinements leur sont attribuables.

Les volcans anciens démantelés par l'érosion (tel le piton des Neiges, à la Réunion) ont perdu leur surface initiale, mais leur structure interne conserve des caractères défavorables au moins dans certaines zones et dans certaines directions ; au contraire au large du centre d'émission des laves, les coulées sont presque horizontales et séparées par des intercalations de scories très perméables, elles fournissent des structures très stables, justifiant les hautes parois très raides des vallées (qui ont mérité le nom de « remparts »).

Les cônes tronqués deviennent plus sensibles à cette action de l'eau souterraine que les cônes "pointus », puisqu'ils sont seuls susceptibles de se « remplir » d'eau souterraine jusqu'au sommet. Certains volcans sont largement tronqués, comme le Kilimandjaro (Tanzanie) ; les cratères peuvent être occupés par un lac, temporaire ou non, ou par une calotte de glace dont la fusion alimente l'eau souterraine.

Enfin, comme tous les grands sommets isolés, les volcans sont la cible d'actions météorologiques intenses, souvent mal identifiées; c'est une raison de plus pour leur instabilité.

\section{Coulées de boue et de débris, lahars}

Divers mouvements de masse sur les flancs des volcans ont des causes proprement volcaniques : nuées ardentes comme celle de 1902 à la montagne Pelée (Martinique) ; bombement précédant I'écroulement du versant comme en 1980 au mont Saint-Helens (Washington) ; échauffement du glacier, fournissant l'eau pour les coulées boueuses du Nevado del Ruiz (en Colombie, 1985) ; sans oublier les coulées de laves et les retombées pyroclastiques.

Mais d'autres causes tiennent à la forme ou à la structure du volcan, et non au volcanisme proprement dit: le débordement de lacs de cratère à Java, sous l'effet de plujes diluviennes, est à l'origine des lahars stricto sensu. Hors du domaine volcanique, d'autres débordements de lacs ont produit aussi des coulées de boue dévastatrices (notamment dans la Cordillère des Andes), comme les débordements de bassins de décantation de stériles miniers (Merriespruit, Afrique du Sud, 1993).

L'avalanche de débris du volcan Ontake a été déclenchée par un séisme, mais la rupture s'explique par la structure en couches parallèles au versant. A l'aval de la rupture, les phénomènes ne sont pas sans analogie avec les avalanches de débris du Huascaran (Pérou, 1962 et 1970), issues d'écroulements rocheux sans rapport avec des structures volcaniques.

Les volcans les plus hauts sont pour cette raison dotés d'une énergie potentielle plus importante, qui assure la progression des coulées à de très grandes distances ; ainsi le mont Rainier, à 4392 m d'altitude a nourri des coulées (préhistoriques) jusqu'au site actuel de la ville de Seattle, à plus de $100 \mathrm{~km}$.

Les trois terrils cités dans l'introduction ont donné naissance à des coulées de boue : cendres industrielles à Jupille, où la coulée a détruit une école (Calembert et Dantinne, 1967) : stériles de mine de charbon à Aberfan (Williams, 1967 ; Duffaut, 1982), la coulée a détruit plusieurs maisons dont encore l'école ; stériles d'une mine métallique à Stava, la coulée descend la vallée et emporte ou submerge plusieurs maisons et hôtels. L'eau est en cause dans ces trois catastrophes bien que les deux premières coulées aient été qualifiées de sèches.

4

\section{Comparaison des reliefs, volcans, tas ou terrils, avec les autres reliefs et les constructions}

Contrairement aux reliefs et aux tas de toute nature, la plupart des constructions sont creuses (bâtiments, usines, y compris clochers, minarets et cheminées) et ne sont pas prévues pour contenir de l'eau (exceptionnellement on a vu à Vaison-la-Romaine le 22 septembre 1992 un mur d'immeuble dominant la rivière à l'aval immédiat du pont romain sur l'Ouvèze céder sous la pression de l'eau entrée à l'intérieur par la porte sur rue); les murs verticaux mettent à profit des liants, dans la maçonnerie ou le béton, ou des matériaux 
tenaces, bois ou métal ; quant aux réservoirs d'eau en superstructure, ils sont toujours en matériaux tenaces ou précontraints (dès les tonneaux).

Parmi les tas et remblais, il faut distinguer ceux qui sont faits pour durer, voire pour supporter des charges, et les tas temporaires, ceux-ci sont souvent implantés sans études ni précautions, ceux-là reçoivent plus ou moins de soins; les remblais de chemins de fer et d'autoroutes, et surtout les barrages en remblai, qu'il s'agisse de terres ou d'enrochements, sont particulièrement soignés. Certains terrils ont été particulièrement négligés, sans aucun traitement du terrain ni drainage sous le remblai ; c'est le cas de la dique de Carling (Moselle), un remblai de voie ferrée privée, posé sur la terre végétale, qui a été pourtant surélevé plus tard pour servir de barrage à un bassin de décantation de cendres, digue rompue en 1980. L'absence de drainage sous les terrils d'Aberfan a été retenue parmi les causes de la catastrophe.

Il va sans dire que les volcans s'installent sur des terrains qui n'ont été ni choisis ni préparés pour les recevoir, donc sans fondation appropriée ni dispositif de drainage ; d'ailleurs les coulées (tant de laves volcaniques que de boue), les dunes, les moraines, envahissent aussi des terrains non préparés.

Avec les dunes, les moraines, les eskers, les cônes d'éboulis et d'alluvions, deltas compris, les volcans font partie des reliefs positifs, reliefs naturels construits (Fig. 4, d'après Duffaut, 1994), par opposition aux reliefs négatifs, reliefs creusés ou découpés (par les agents d'érosion) dans une masse antérieure (reliefs $\alpha$ sculptés » de Thérond, d'après Besson, 1996); les escarpements de faille échappent à cette classification. Bien davantage que les autres catégories de reliefs positifs. les volcans sont construits par superposition de matériaux très variés, non sans périodes intermédiaires d'altération (voire de ravinement et d'érosion) entre les apports successifs. Au contraire, les dunes sont relativernent homogènes (sable fin), les éboulis et les alluvions un peu moins, ceux-ci plus exposés que dunes et éboulis á la montée de l'eau souterraine ; les moraines se singularisent par leur forte hétérométrie et parfois des phases de compactage (notamment pour les moraines de fond appelées en anglais tills).

Certains matériaux des volcans sont favorables à la stabilité : les fragments encore visqueux se ressoudent et leur accumulation donne des roches à grande cohésion (malgré une faible densité due à leur teneur en bulles), la plupart sont au contraire défavorables : en particulier les cendres fines sont très altérables, et les argiles d'altération ont une perméabilité très faible.
Si la structure interne des volcans est largement aléatoire (chaotique ?), elle se conforme toutefois à un modèle de cônes emboîtés, avec des surfaces de séparation inclinées vers l'extérieur, c'est-à-dire dans la direction la plus défavorable. Les ravinements entre phases de construction, qu'ils proviennent de l'écoulement des pluies, de la fonte des neiges, du débordement d'un lac de cratère ou des glissements de terrain survenus avant les dernières additions de matériaux, sont susceptibles de fournir des voies priviléqiées à la circulation interne de l'eau.

Enfin beaucoup de volcans sont isolés, et constituent donc des reliefs remarquables, certains de grande hauteur $(3,5 \mathrm{~km}$ pour le Fuji-Yama, 4 pour le mont Rainier, 10 pour le Mauna Loa, en comptant depuis le plancher océanique). Parmi les autres reliefs naturels on trouve peu cle sommets isolés comparables par la hauteur, mais il en est de plus élancés : dans ses dessins, Alexandre de Humboldt (1814) avait un peu exagéré les pentes des volcans des Andes (qui sont pourtant parmi les plus pentus au monde), mais aucun n'approche les pyramides ou aiguilles des pics rocheux extrêmes, tous sculptés par l'érosion glaciaire, le Mitre Peak en Nouvelle-Zélande, le Stetinden en Norvège, le Cervin à la frontière italo-suisse (Duffaut, 1993), et surtout les aiguilles des Andes de Patagonie, Paine et Cerro Torre.

\section{5}

\section{Conclusion}

Les flancs des volcans sont, davantage que beaucoup d'autres reliefs, exposés à des mouvements de masse dangereux et souvent catastrophiques, non seulement en raison de phénomènes proprement voicaniques, mais parce que leur structure interne comporte des surfaces de séparation inclinées vers l'extérieur. Parmi celles-ci, les couches de cendres, par leur évolution vers des argiles, fournissent des contrastes de perméabilité sur lesquelles se concentrent les forces exercées par l'eau souterraine.

Après chaque accident de terril les autorités se préoccupent de mieux comprendre les phénomènes; le cas échéant des règles plus strictes sont établies pour leur édification et leur surveillance. Une meilleure compréhension des analogies et différences entre terrils et volcans pourrait aider à la prévention et à la prévision des ruptures aux flancs des volcans, qu'ils soient actifs ou considêrés comme éteints.

\section{$\overline{\text { Bibliographie }}$}

Besson L. - Les risques naturels en montagne. Publi-Artes, 1996.

Calembert L., Dantinne R. - ir L'avalanche de cendres volantes survenue à Jupille le 3 février 1961 n. Volume d'hommage au professeur Campus, Liège, 1967. p. $41-57$.

Duffaut P., Louis C. - " L'eau souterraine et J'équilibre des pentes naturelles $n, 24^{\circ}$ Congrès géologique international, Montréal, section 13, 1972, p. 107-116; et Bulletin du BRGM, $2^{r}$ série $111 n^{\circ} 4$, p. 3-12.
Duffaut P. - « La rupture du terril d'Aberfan (pays de Galles) le 21 octobre 1966 D. Revue de l'industrie minérale, 1982. p. $413-418$.

Duffaut P. - - Sếisme et glissements dé terrain au mont Ontake (Japon) x. Annales ITBTP 457, 1987, p. 47-69.

Duffaut $P$. - « Any geotechnical engineer has to face groundwatern. Colloque Porous or fractured unsaturated media: transport and behaviour. École polytechnique fédérale de Lausanne, 1992. p. 391-398.
Duffaut P. - " Sur la stabilité des pentes et des pics $n$. Revue française de géotechnique $n^{\circ} 63,1993$, p. $57-64$.

Duffaut P. - " Stabilité des barrages et des pentes naturelles v. Congrès international des grandis barrages. Durban, vol.IV, 1994. p. 809-822.

Humboldt A. (de) - Relation historique du voyage aux régions équinoxiales du Nouveau Continent. Paris, 1814.

Williams G.M.J. - « Inquiry into the Aberfan disaster x. His Majesty Stationery Office, Londres, 1967. 